\title{
Multichannel photodiode detector for ultrafast optical spectroscopy
}

\author{
T. Mertelj, , 田 N.Vujičić, ${ }^{1,2}$ T. Borzda, ${ }^{1,3}$ I. Vaskivskyi, ${ }^{1}$ A. Pogrebna, ${ }^{1,3}$ and D. Mihailovic ${ }^{1,3,4}$ \\ ${ }^{1}$ Complex Matter Dept., Jozef Stefan Institute, Jamova 39, Ljubljana, SI-1000, Ljubljana, Slovenia \\ ${ }^{2}$ Institute of Physics, Bijenička 46, HR-10000 Zagreb, Croatia \\ ${ }^{3}$ Jožef Stefan International Postgraduate School, Jamova 39, SI-1000 Ljubljana, Slovenia \\ ${ }^{4}$ CENN Nanocenter, Jamova 39, Ljubljana SI-1000, Slovenia
}

(Dated: August 22, 2018)

\begin{abstract}
Construction and characterization of a multichannel photodiode detector based on commercially available components with high signal to noise of $\sim 10^{6}$ and a rapid frame rate, suitable for time resolved femtosecond spectroscopy with high repetition femtosecond sources, is presented.
\end{abstract}

\section{INTRODUCTION}

In recent years femtosecond spectroscopy has been established as a standard tool to investigate physics of novel solid-state materials such as nonconventional superconductors, electronic crystals and related collectively ordered systems. 1-25] Majority of the published work was done using narrowband optical probe pulses. There are several reasons for use of narrowband probes. Apart from simplicity of a setup, the main reason is the necessity to use lockin modulation techniques to reject the laser noise in order to detect small changes in optical responses. On the other hand, use of broadband optical pulses [20, 23 26] can provide additional spectral information which is time consuming to obtain by means of wavelength-tunable narrowband optical pulses [9, 27, 28].

Since many low temperature ordered states are easily destroyed by rather weak photoexcitation. 15, 27, 29, 30. the energy of the probe pulses must be kept low enough. In the case of broadband probe pulses this requires very efficient multichannel detection system that is fast enough to enable employment of the lockin-like modulation techniques [31, 32] to suppress the laser noise. Such systems have been implemented recently in a $\mathrm{kHz}$ system [31] and cavity dumped fast repetition system 33. with the signal to noise ratio (SNR) $\sim 10^{4}$ enabling detection of $>10^{-4}$ photoinduced relative changes of optical constants.

Here we present construction and characterization of a multichannel photodiode detection system built from commercially available components with SNR $>10^{5}-10^{6}$ enabling lockin-like modulation techniques with modulation frequency up to $3 \mathrm{kHz}$ appropriate for experiments using high repetition $(\gg 1 \mathrm{kHz})$ pulsed laser sources.

\section{STATEMENT OF THE PROBLEM}

In time resolved pump-probe experiments a relatively small changes of the reflectivity or transmittance of the

\footnotetext{
* tomaz.mertelj@ijs.si
}

sample are measured,

$$
\Delta G_{j} / G_{j}=\Delta I_{j} / I_{j}
$$

where $G$ stands either for reflectivity, $R_{j}$, or transmittance, $T_{j}$, and $I_{j}$ represents the light intensity in the channel $j$. In a photodetector the SNR of a single channel is fundamentally limited by the number of acquired photoelectrons, $n_{\mathrm{pe}}$. For $\Delta G_{j} / G_{j} \sim 10^{-6}$ the $\mathrm{SNR}=10^{6}$ is required. In the shot-noise limit $n_{\mathrm{pe}}=10^{12}$ photoelectrons need to be acquired corresponding to a charge of $q=160 \mathrm{nC}$. Assuming 100\% quantum efficiency, this corresponds to $\sim 250 \mathrm{~nJ}$ of optical energy (at 800-nm photon wavelength) per channel. Taking a typical convenient laser spot size 34 of $\sim(100 \mu \mathrm{m})^{2}$ this leads to $\sim 2.5 \mathrm{~mJ} / \mathrm{cm}^{2}$ of reflected/transmitted optical energy per channel. Limiting the single probe pulse fluence to 10 $\mu \mathrm{J} / \mathrm{cm}^{2}$ and assuming 50 channels the required number of probe pulses is $\sim 10000$ resulting in the minimum $\sim 10$ $\mathrm{s}$ acquisition time per spectrum for a $\mathrm{kHz}$ laser system. With high repetition lasers this time can be considerably shorter, below one second. The SNR $=10^{6}$ is therefore feasible, provided that noise from other sources, especially the laser source noise, can be eliminated.

Modern spectroscopic CCDs have output register well capacity of $\sim 10^{6}(q \sim 0.16 \mathrm{pC})$ electrons and can achieve $\sim 1 \mathrm{kHz}$ readout rate (see for example ANDOR datasheets) leading to impractical [35] accumulation times of 36 ] $>100 \mathrm{~s}$ per spectrum. Commercially available CMOS and NMOS linear image sensors, on the other hand, offer on-chip effective well capacities in excess of $10 \mathrm{pC}$ (see for example Hammamatsu data sheets), but mainly offer sequential readout only, which introduces dead-time and exposure-control problems 37 when used in conjunction with high repetition lasers.

Recently, a custom CMOS detector was presented, 32] incorporating four independent storage capacitors per pixel with the effective well capacity of $600 \cdot 10^{6}(q \sim 100$ $\mathrm{pC}$ ) photoelectrons, capable of operating with up to $\sim 40$ $\mathrm{kHz}$ frame rate, enabling a multichannel pseudo-lockin detection at up to $\sim 10 \mathrm{kHz}$ modulation frequency. To our best knowledge this detector is not yet commercially available so a similar solution based on commercially available components is desirable. 


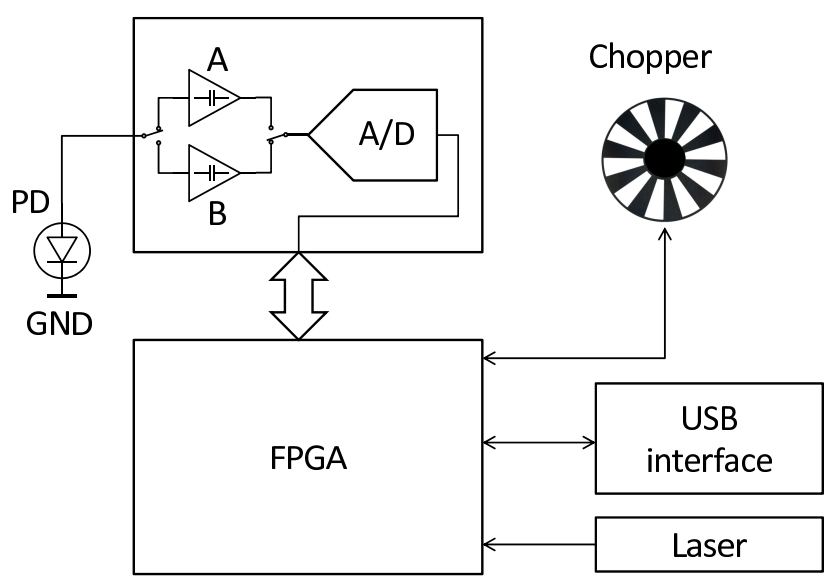

Figure 1. A schematic diagram of the detector. PD - photodiode (one channel), GND - ground, A, B - integrators. A/D analog to digital converter, FPGA - fully programmable gate array.

\section{OUR SOLUTION}

Our solution is based on a multichannel Texas Instruments DDC-series current-input analog-to-digital (A/D) converter, that offers up to $150 \mathrm{pC}$ effective well capacity with up to $6.25 \mathrm{kHz}$ frame rate. Thus, $n_{\mathrm{pe}}=10^{12}$ photoelectrons can be accumulated on a timescale of second. Due to the dual switched integrator front-end the integration window of each input is synchronous, enabling precise exposure control with almost no dead time, the condition which is required in the case of high repetition lasers.

Different types of photodiode arrays can be coupled to the $\mathrm{AD}$ converter. We chose a 46-channel Hamamatsu S4111 series silicon photodiode array and a 16-channel Hamamatsu G7150-16 InGaAs PIN photodiode array for visible and near infrared spectral regions, respectively. The rather small number of channels is not a disadvantage since the high SNR requirement together with the probe pulse energy limitation inherently constrain the channel number.

The digital interface to the $\mathrm{AD}$ converter (see Fig. 11) was implemented in a field programmable gate array (FPGA) providing control logic, clocks, synchronization with a chopper and laser, frame averaging as well as data transfer to an USB interface circuit.

Since for transient reflectivity/transmittance measurements a full phase sensitive detection is not necessary two spectra are acquired during each pump modulation period. The photoinduced intensity is calculated by,

$$
\Delta I_{j} / I_{j}=\frac{I_{j, \text { on }}-I_{j, \text { off }}}{I_{j, \text { off }}},
$$

where $I_{j, \text { on }}$ and $I_{j \text {,off }}$ correspond to intensities measured during opening and blocking periods of the chopped
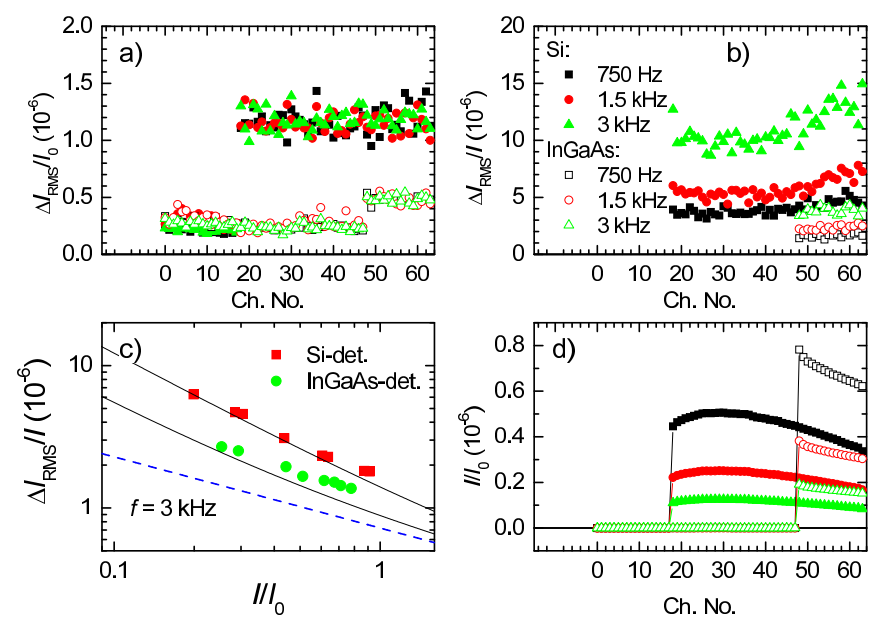

Figure 2. (a) The non-illuminated-detector noise floor at different modulation frequencies. (b) The signal-noise floor measured under CW halogen-lamp illumination. The corresponding intensities are shown in (d). The non uniformity is due to nonhomogenoeus illumination. (c) The CW-illumination noise floor as a function of intensity. The blue dashed line is the shot-noise floor while the thin black lines are the calculated total noise taking into account both, the dark noise from (a) and the shot noise, with no fitting parameters. At each measurement 2000 samples were averaged, corresponding to the total available photoelectron charge capacity per channel of, $q_{\max }=300 \mathrm{nC}$. $I_{0}$ is the saturation intensity at the full effective-well capacity of $q_{0}=150 \mathrm{pC}$ per sample. Only 48 channels (ch. no. 19 to 63) in the case of Si photodiode array and 16 channels (ch. no. 49 to 63) in the case of InGaAs photodiode array were connected.

pump beam, respectively. To avoid exposure during the integrator switching time the detector and chopper are synchronized to the laser source by the FPGA circuitry. 38]

\section{RESULTS}

In Fig. 2 we show results of a basic characterization of the detector. In addition to the photoelectron shot noise the integrators input noise, the photodiode-capacitance thermal noise and other electronic noise picked from environment are present, while the digitization noise should be negligible with respect to the shot-noise due to the 20-bit resolution of the on-chip ADCs.

In Fig. 2 (a) we show the dark noise of the detector measured under typical experimental conditions by averaging 2000 readouts corresponding to $0.6-2 \mathrm{~s}$ total integration time per single spectrum. The unconnected channels[39] show noise of $\sim 3 \cdot 10^{-7} I_{0}$, where $I_{0}$ corresponds to the saturation intensity at the full effective-well capacity of $q_{0}=150 \mathrm{pC}$. The connected channels show additional noise of $\sim 1.2 \cdot 10^{-6} I_{0}$ and $\sim 0.5 \cdot 10^{-6} I_{0}$ for the $\mathrm{Si}$ and InGaAs detectors, respectively. 
a) $1 \mathrm{~T}-\mathrm{TaS}_{2}$

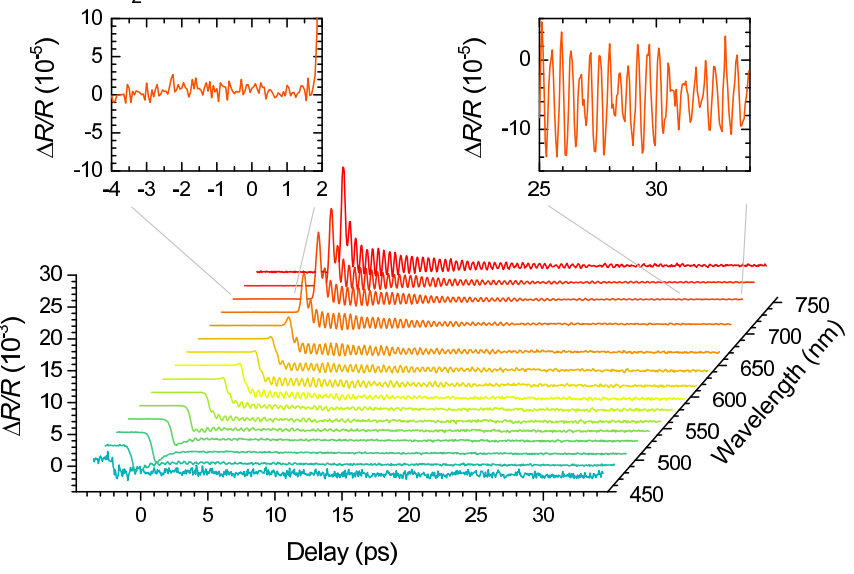

b) Al-Ni-Co quasicrystal

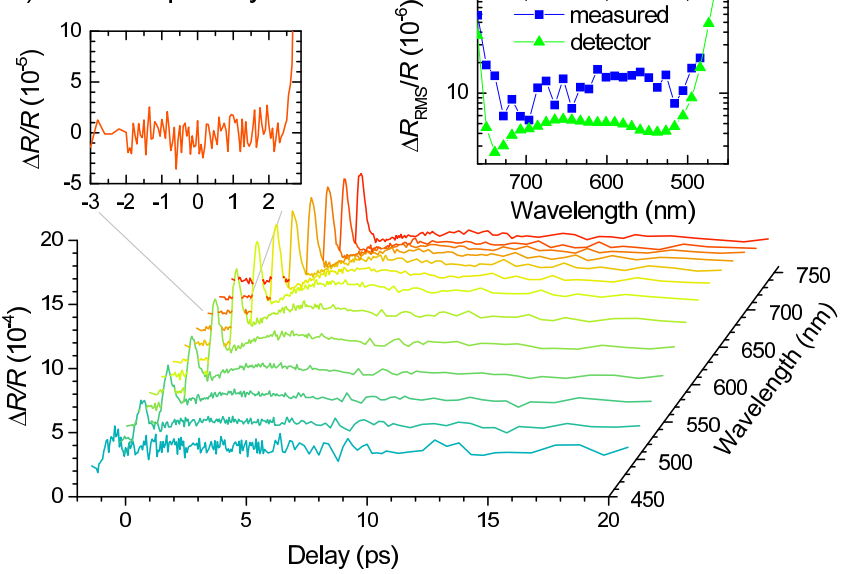

Figure 3. a) The transient optical reflectivity of $1 T-\mathrm{TaS}_{2}$ at $120 \mathrm{~K}$. The RMS noise level in the least noisy region is of order of $10^{-5}$ as shown in the left panel so the weak coherent oscillations shown in the right panel can be observed at long delays. b) The transient optical reflectivity of an Al-Ni-Co quasicrystal [16] at room temperature. The noise level in the least noisy region is shown in the left panel. The wavelength dependence of the RMS noise level is shown in the right panel. The detector noise was estimated from the unilluminated detector data. The chirp present in the supercontinuum probe pulses can be clearly seen in both cases.

The CW-illuminated-detector 40 noise at different modulation frequencies is shown in Fig. 2 (b). Due to the fixed number of readouts (2000) the total integration time and consequently the total photelectron counts are inversely proportional to the modulation frequency, $f$, resulting in a higher signal noise floor at higher modulation frequencies.

In Fig. 22 (c) we show the illumination-intensity dependence of the signal-noise floor at $f=3 \mathrm{kHz}$. The measured signal-noise floor is well described by the combi- nation of the intensity-independent dark-noise from Fig. 2 (a) and the shot noise floor shown in Fig. 2 (c) with the blue dashed line. It can be seen that the detector is capable of operation near the shot-noise limit with the signal-noise floor of $\sim 2 \cdot 10^{-6}$ at $\sim 1$ s acquisition time.

In Fig. 3 we show two actual data examples measured by the Si photodiode array, where the visible broadband supercontinuum pulses were generated [41] in a $2.5-\mathrm{mm}$ thick sapphire window from $800-\mathrm{nm} \sim 0.5-\mu \mathrm{J} 50$-fs pulses from a high repetition rate optical regenerative amplifier. The IR part of the supercontinuum $(\lambda>\sim 750$ $\mathrm{nm})$ was rejected by a combination of Shott glass and interference filters. The reflected probe beam was coupled to an in-house built 300-mm focal-length spectrometer by means of a $0.6-\mathrm{mm}$ core-diameter fiber. A 300 grooves $/ \mathrm{mm}$ transmissive optical grating was chosen to maximize throughput in the $800-400 \mathrm{~nm}$ spectral region.

The total integration time for each delay was $\sim 2$ s resulting in $\sim 30$ min acquisition time for the case of the coherent oscillations [7] in $1 T-\mathrm{TaS}_{2}$ [Fig. 3 (a)] with 760 delay points. Using $150 \mathrm{pC}$ effective-well depth we obtained the RMS noise floor of $<10^{-5}$ in the least noisy part of the spectrum (see Fig. 4). The most significant contribution to the noise floor is clearly not due to the shot noise and the detector noise but due to the supercontinuum source noise, which strongly increases at shorter wavelengths.

A smaller effective-well depth of $50 \mathrm{pC}$ was used for measurement in an Al-Ni-Co quasi-crystal [16] together with a lower supercontinuum probe-pulse energy of $\sim$ $170 \mathrm{pJ}$ per pulse, resulting in $\sim 2 \mu \mathrm{J} / \mathrm{cm}^{2}$ fluence with $(100 \mu \mathrm{m})^{2}$ probe spot size. Due to the lower probe-pulse energy the noise floor is somewhat larger $\left(\sim 2 \cdot 10^{-5}\right)$ as shown in Fig. 3 (b).

\section{CONCLUSIONS}

A high signal-to-noise $\left(\sim 10^{6}\right)$ multichannel photodetector for femtosecond transient optical spectroscopy in visible and NIR was constructed from commercially available components. The $6-\mathrm{kHz}$ synchronous sampling rate enables lockin-like modulation detection to suppress the optical source noise enabling $<10^{-5}$ transient reflectivity/transmittance RMS signal-noise floor in a broad spectral range as shown by two example measurements.

\section{ACKNOWLEDGMENTS}

Work at Jozef Stefan Institute was supported by ARRS (Grant No. P1-0040).

[1] S. V. Chekalin, V. M. Farztdinov, V. V. Golovlyov, V. S. Letokhov, Y. E. Lozovik, Y. A. Matveets, and A. G.

Stepanov, Phys. Rev. Lett. 67, 3860 (1991) 


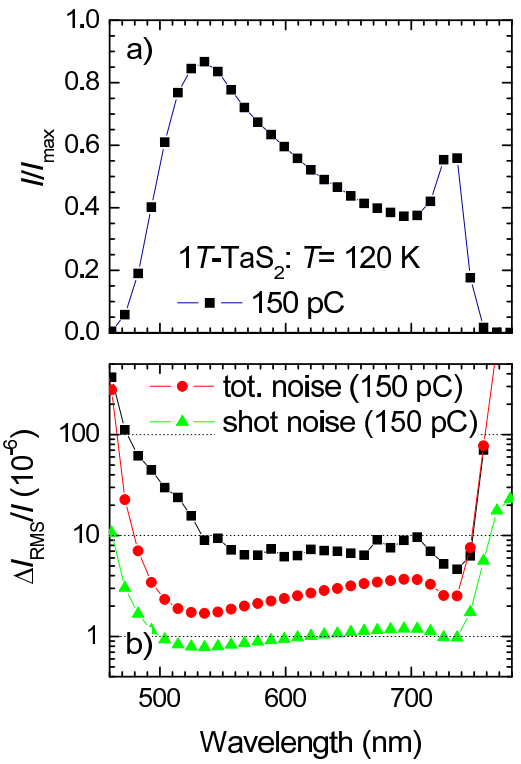

Figure 4. (b) The RMS signal-noise floor for the case of coherent oscillations measurement in $1 T-\mathrm{TaS}_{2}$. The full circles (red) and full triangles (green) correspond to the estimated total detector noise and the shot noise, respectively. The corresponding spectral dependence of the photodiode current is shown in (a).

[2] J. Demsar, B. Podobnik, V. V. Kabanov, T. Wolf, and D. Mihailovic, Phys. Rev. Lett. 82, 4918 (1999).

[3] J. Demsar, K. Biljaković, and D. Mihailovic, Phys. Rev. Lett. 83, 800 (1999)

[4] A. I. Lobad, A. Taylor, C. Kwon, S. Trugman, and T. Gosnell, Chemical Physics 251, 227 (2000)

[5] R. Kaindl, M. Woerner, T. Elsaesser, D. Smith, J. Ryan, G. Farnan, M. McCurry, and D. Walmsley, Science 287, 470 (2000).

[6] D. Dvorsek, V. V. Kabanov, J. Demsar, S. M. Kazakov, J. Karpinski, and D. Mihailovic, Phys. Rev. B 66, 020510 (2002)

[7] J. Demsar, L. Forró, H. Berger, and D. Mihailovic, Phys. Rev. B 66, 041101 (2002)

[8] G. P. Segre, N. Gedik, J. Orenstein, D. A. Bonn, R. Liang, and W. N. Hardy, Phys. Rev. Lett. 88, 137001 (2002).

[9] S. Tomimoto, S. Miyasaka, T. Ogasawara, H. Okamoto, and Y. Tokura, Phys. Rev. B 68, 035106 (2003).

[10] R. A. Kaindl, M. A. Carnahan, D. S. Chemla, S. Oh, and J. N. Eckstein, Phys. Rev. B 72, 060510 (2005)

[11] N. Gedik, J. Orenstein, R. Liang, D. Bonn, and W. Hardy, Science 300, 1410 (2003).

[12] R. P. Prasankumar, H. Okamura, H. Imai, Y. Shimakawa, Y. Kubo, S. A. Trugman, A. J. Taylor, and R. D. Averitt, Phys. Rev. Lett. 95, 267404 (2005).

[13] R. V. Yusupov, T. Mertelj, J.-H. Chu, I. R. Fisher, and D. Mihailovic, Phys. Rev. Lett. 101, 246402 (2008)

[14] Y. H. Liu, Y. Toda, K. Shimatake, N. Momono, M. Oda, and M. Ido, Phys. Rev. Lett. 101, 137003 (2008).

[15] C. Giannetti, G. Coslovich, F. Cilento, G. Ferrini, H. Eisaki, N. Kaneko, M. Greven, and F. Parmigiani,
Phys. Rev. B 79, 224502 (2009).

[16] T. Mertelj, A. Ošlak, J. Dolinšek, I. R. Fisher, V. V. Kabanov, and D. Mihailovic, Phys. Rev. Lett. 102, 086405 (2009).

[17] T. Mertelj, V. Kabanov, C. Gadermaier, N. Zhigadlo, S. Katrych, J. Karpinski, and D. Mihailovic, Physical Review Letters 102, 117002 (2009).

[18] H. Schäfer, V. V. Kabanov, M. Beyer, K. Biljakovic, and J. Demsar, Phys. Rev. Lett. 105, 066402 (2010).

[19] D. H. Torchinsky, G. F. Chen, J. L. Luo, N. L. Wang, and N. Gedik, Phys. Rev. Lett. 105, 027005 (2010)

[20] C. Gadermaier, A. S. Alexandrov, V. V. Kabanov, P. Kusar, T. Mertelj, X. Yao, C. Manzoni, D. Brida, G. Cerullo, and D. Mihailovic, Phys. Rev. Lett. 105, 257001 (2010).

[21] B. Mansart, D. Boschetto, A. Savoia, F. RullierAlbenque, F. Bouquet, E. Papalazarou, A. Forget, D. Colson, A. Rousse, and M. Marsi, Phys. Rev. B 82, 024513 (2010).

[22] P. Kusar, T. Mertelj, V. V. Kabanov, J.-H. Chu, I. R. Fisher, H. Berger, L. Forró, and D. Mihailovic, Phys. Rev. B 83, 035104 (2011).

[23] C. Giannetti, F. Cilento, S. Dal Conte, G. Coslovich, G. Ferrini, H. Molegraaf, M. Raichle, R. Liang, H. Eisaki, M. Greven, et al., Nature Communications 2, 353 (2011).

[24] K. W. Kim, A. Pashkin, H. Schäfer, M. Beyer, M. Porer, T. Wolf, C. Bernhard, J. Demsar, R. Huber, and A. Leitenstorfer, Nature Materials 11, 497 (2012).

[25] B. Mansart, M. J. G. Cottet, T. J. Penfold, S. B. Dugdale, R. Tediosi, M. Chergui, and F. Carbone, Proceedings of the National Academy of Sciences 109, 5603 (2012) http://www.pnas.org/content/109/15/5603.full.pdf+html

[26] A. Pogrebna, N. Vujičić, T. Mertelj, T. Borzda, G. Cao, Z. A. Xu, J.-H. Chu, I. R. Fisher, and D. Mihailovic, Phys. Rev. B 89, 165131 (2014).

[27] T. Ogasawara, T. Kimura, T. Ishikawa, M. Kuwata-Gonokami, and Y. Tokura, Phys. Rev. B 63, 113105 (2001).

[28] H. Okamoto, T. Miyagoe, K. Kobayashi, H. Uemura, H. Nishioka, H. Matsuzaki, A. Sawa, and Y. Tokura, Phys. Rev. B 82, 060513 (2010).

[29] P. Kusar, V. Kabanov, J. Demsar, T. Mertelj, S. Sugai, and D. Mihailovic, Physical Review Letters 101, 227001 (2008).

[30] R. V. Yusupov, T. Mertelj, P. Kusar, V. Kabanov, S. Brazovskii, J.-H. Chu, I. R. Fisher, and D. Mihailovic, Nature Physics 6, 681 (2010).

[31] D. Polli, L. LÃCer, and G. Cerullo, Review of Scientific Instruments 78, 103108 (2007)

[32] R. J. Smith, R. A. Light, S. D. Sharples, N. S. Johnston, M. C. Pitter, and M. G. Somekh, Review of Scientific Instruments 81, 024901 (2010)

[33] F. Cilento, C. Giannetti, G. Ferrini, S. Dal Conte, T. Sala, G. Coslovich, M. Rini, A. Cavalleri, and F. Parmigiani, Applied Physics Letters 96, 021102 (2010).

[34] The spot size is often limited by the sample size.

[35] To measure time evolution spectra at different delays usually requires several tens of spectra per scan.

[36] Here we assume that by a proper optical setup $\sim 10$ columns can be joined into a single spectral channel leading to an effective $\sim 100$ channel detector.

[37] With a $\mathrm{kHz}$ laser a single optical pulse per scan, which is well defined in time, implements its own shutter. With 
high repetition laser an external shutter is needed to prevent exposure during the readout.

[38] For the cases presented here a high repetition $(250 \mathrm{kHz})$ optical regenerative amplifier (Coherent REGA) was employed.
[39] Channels 0 to 18 for the Si detector and 0 to 48 for InGaAs detector.

[40] A $20 \mathrm{~W}$ halogen lamp driven by a stabilized DC power supply was used as a source.

[41] M. Bradler, P. Baum, and E. Riedle, Applied Physics B 97, 561 (2009). 